\title{
Be Phenomena in Young Early-type Stars: Various Forms of Interaction between the Star and the Circumstellar Gaseous Envelope
}

\author{
M. Pogodin \\ Pulkovo Observatory, 196140 St. Petersburg, Russia
}

\begin{abstract}
New results of high-resolution spectroscopy of four pre-main sequence $\mathrm{Ae} / \mathrm{Be}$ stars are presented. An analysis of parameters of lines originating in different regions of the circumstellar (CS) envelope ( $\mathrm{H} \alpha$, $\mathrm{H} \beta$, He I 5876, DNaI) allows to reconstruct a picture of the interaction between the star and the CS environment which can be displayed in different forms. At least two separate processes seem to impact the structural and kinematical properties of the envelope: the stellar wind from the stellar surface and the matter infall onto the star from the CS media. A possible relation between these two phenomena is discussed in the framework of different models. Some similarity between observational phenomena in Herbig $\mathrm{Ae} / \mathrm{Be}$ and classical Be stars is noted in spite of their difference in evolutionary status.
\end{abstract}

The Herbig Ae/Be stars (HAEBESs) are believed to be pre-main sequence (PMS) objects of intermediate mass $2 \mathrm{M}_{\odot}<M<10 \mathrm{M}_{\odot}$ (Herbig 1960, Finkenzeller \& Mundt 1984, Thé et al. 1994). Extensive studies of these stars during the last three decades led to the discovery of many of their properties. Now it is known that HAEBESs: a) are surrounded by extended dust/gas envelopes with an inhomogeneous structure, b) possess high-temperature zones near the stellar surface, c) display signs of stellar wind as well as matter infall onto the star. Nevertheless, a number of problems are still to be solved concerning: a) the sources of the observed activity, b) the origin of high-temperature regions of circumstellar (CS) gas, c) the driving mechanisms of stellar wind, d) the role of magnetic fields in the general picture of observed phenomena.

Two possible models of star/CS interaction are used to explain the spectroscopic behaviour of four HAEBESs: HD 259431 (B5Vep), HD 37806 (B9Ve), HD 200775 (B2/3ep), and HD 31648 (A2/3ep).

The first phenomenological model was suggested by Grinin \& Rostopchina (1996). This model is an attempt to develop the classical accretion magnetospheric (AM) model which is believed for the pre-main sequence low mass objects T Tau-type stars (Camenzind 1990, Königl 1991, Hartmann et al. 1994, Shu et al. 1994). According to the model the accreting gas in a fragmented gas-dust disk does not arrive at the stellar surface but is completely ejected in the form of a wind after its interaction with the magnetosphere (so called propeller-effect). In spite of the fact that the model can explain many observational properties of HAEBESs, some phenomena are hard (or impossible at all) to be interpreted in 
its framework: a) a global change of the envelope kinematics which is episodically observed in some objects (Beskrovnaya et al. 1991, 1994; Finkenzeller \& Mundt 1984; Pogodin 1992; Shevchenko et al. 1994; Vieira et al. 1999); and b) different observational behaviour of HAEBEs depending on the spectral class. Now it is found that a homogeneous subgroup of B8-A2 objects with strong stellar winds exists among heterogeneous HAEBESs.

An alternative type of HAEBE model can be proposed which resembles in some extent the model of a classical Be star. According to the model: a) the rotating disk and the wind are the two main originally independent components of the CS gaseous envelope around a HAEBE; b) the disk is concentrated to the equatorial plane, whereas the wind possesses a wider latitudinal distribution, although likely to be limited in latitude; c) the wind is a more active factor determining the conditions in the envelope. It originates inside the star and can vary in rate and can be inhomogeneous in latitude and azimuth.

The high-resolution $(R \sim 30000)$ spectroscopic observations of four programme stars were carried out in $1997-1998$ with the $2.6 \mathrm{~m}$ Shajn telescope of the Crimean Astrophysical Observatory ( $\mathrm{CrAO}$ ) equipped with a coudéspectrograph. The method used was based on a comparative analysis of observational characteristics of lines originating in different regions of CS gas $\mathrm{H} \alpha$, $\mathrm{H} \beta, \mathrm{He}$ I 5876, and the $\mathrm{Na} I$ lines.

The results of the analysis show that the bulk of the CS gas near HD 259431 is likely to be concentrated in the form of a rotating disk without prominent interaction with the star. The He I 5876 emission is more probably connected with the $\operatorname{star}\left(V \sin i=90 \pm 8 \mathrm{~km} \mathrm{~s}^{-1}\right.$, Böhm \& Catala 1995) rather than with the CS disk. This is evidence in favour of the assumption about a chromospheric nature of the emission.

The spectroscopic behaviour of HD 37806 is typical for objects with a strong stellar wind like $\mathrm{AB}$ Aur, HD 163296, etc, but the wind of this star is more stable and not so intense.

The kinematical situation in the envelope of HD 200775 was different in three observing seasons (Fig. 1, left). In March, 1997 the $\mathrm{H} \alpha$ profile demonstrated signs of outflow. It looked as a single profile with a noticeable blueshifted depression. In 1998 the profile was double-peaked with the central absorption being redshifted in March and at zero velocity in October. The profile of He I 5876 absorption was clearly asymmetric in March, 1997 when the signs of wind were observed in $\mathrm{H} \alpha$. This asymmetry can be explained as due to the formation of an additional narrow blue-shifted emission in the velocity range from -100 to $+50 \mathrm{~km} \mathrm{~s}^{-1}$. The origin of this feature can be connected with the chromosphere near the stellar surface $\left(V \sin i=40 \pm 4 \mathrm{~km} \mathrm{~s}^{-1}\right)$ and the base of the wind whose signs are observed in $\mathrm{H} \alpha$. According to Miroshnichenko et al. (1998), such activity of HD 200775 connected with a global kinematical reconstruction of the CS envelope may be cyclic with a period of 1345 days.

In the case of HD 31648 a detailed picture of wind strengthening was obviously observed on October 20-25, 1998 (Fig. 1, right). The latitudinal distribution of the wind zone became more extended, and the mass-loss rate increased during several days. A possible connection between the wind strengthening and the appearance of the red He I 5876 emission on October 21 can be a matter of forthcoming discussion. The appearance of local high-velocity positionally sta- 

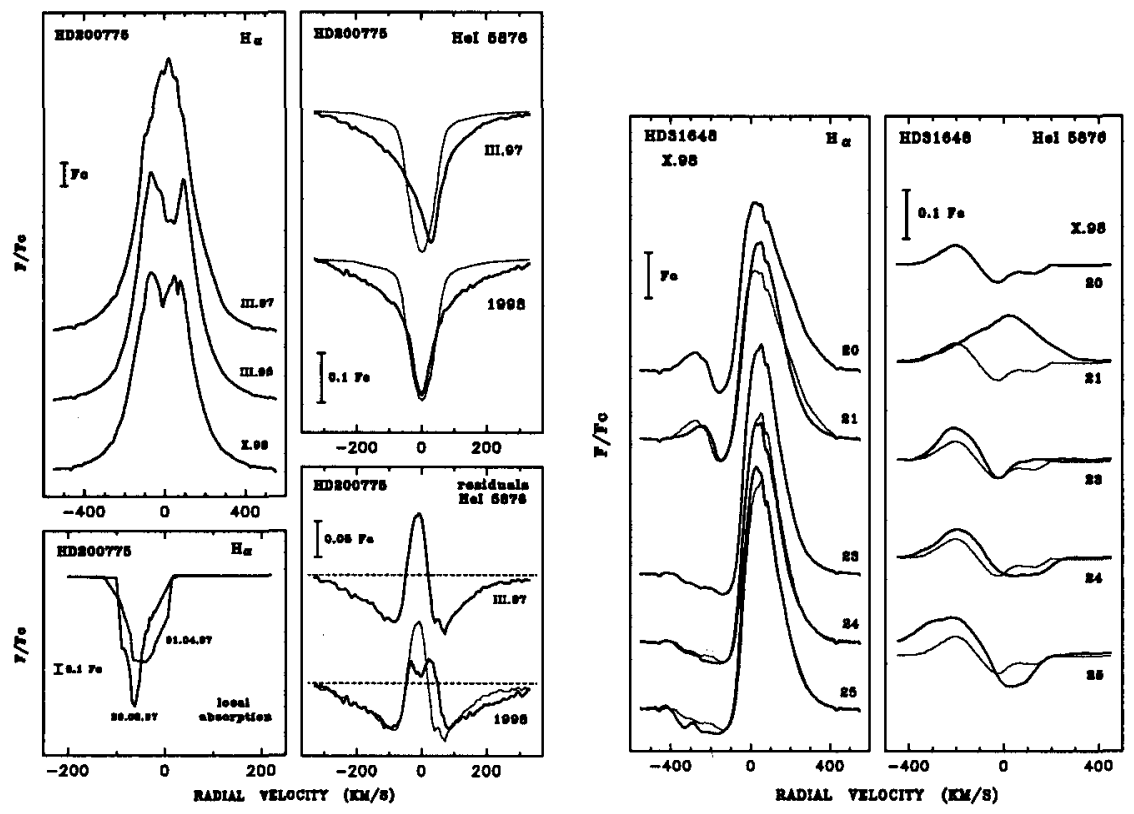

Figure 1. HD 200775 (left panels):

Left (upper): Mean $\mathrm{H} \alpha$ profile in the spectrum of $\mathrm{HD} 200775$ displayed in different observing seasons.

Left (lower): Profile of the blueshifted depression separated from the emission H $\alpha$ profile of HD 200775 observed in March, 1997.

Right (upper): Mean He I 5876 profile in the spectrum of HD 200775 averaged over the data obtained in March-April, 1997 and the whole period of 1998. The synthetic photospheric profile taken from Böhm \& Catala (1995) is given as a dotted line for comparison.

Right (lower): The same as the upper right panel but for residuals constructed relative to the photospheric profile.

HD 31648 (right panels):

The $\mathrm{H} \alpha$ (left) and He I 5876 (right) profiles of HD 31648 obtained on different dates in October, 1998. In the left panel the dotted lines show the $\mathrm{H} \alpha$ profile of October 20 (superimposed for comparison on the profile of October 21) and the profile of October 23 in comparison with profiles of October 24 and 25. In the right panel the He I 5876 profile obtained on October 20 is also repeatedly presented as a dotted line for comparison. 
ble features in the $\mathrm{H} \alpha$ profile when the wind became stronger could be connected with the formation of latitudinal layered structures (LLS) of the wind.

The results of a spectroscopic investigation of four new objects of the programme showed that the model with the wind originating in the star and possessing variable characteristics (density, velocity, latitudinal distribution) is more preferable for the HAEBEs than the AM-type model. The following evidence can be mentioned: a) signs of chromospheric activity in HD 259431 and HD 200775; b) change of CS kinematics in HD 200775; and c) variations of latitudinal distribution of the wind (HD 31648).

In terms of this model the four programme objects demonstrate different cases of star-envelope interaction depending on the strength of the stellar wind.

\section{References}

Beskrovnaya, N.G., Pogodin, M.A., Shcherbakov, A.G., Tarasov, A.E. 1991, Sov. Astron. Lett. 17, 349

Beskrovnaya, N.G., Najdenov, I.D., Pogodin, M.A., Romanyuk, I.I. 1995, A\&A 298,585

Böhm, T., Catala, C. 1995, A\&A 301, 155

Camenzind, M. 1990, Rewiews in Modern Astronomy 3: Accretion and Winds (Springer, Berlin), 234

Finkenzeller, U., Mundt, R. 1984, A\&AS 55, 109

Grinin, V.P., Rostopchina, A.N. 1996, Astron. Reports 40, 171

Hartmann, L., Hewett, R., Calvet, N. 1994, ApJ 426, 669

Herbig, G.H. 1960, ApJS 4, 337

Königl, A. 1991, ApJ 370, L39

Miroshnichenko, A.S., Bjorkman, K.S., Mulliss, C.L. et al. 1998, PASP 110, 883

Pogodin, M.A. 1992, Sov. Astron. Lett. 18, 437

Shevchenko, V.S., Grankin, K.N., Ibragimov, M.A., et al. 1994, ASP Conference Series 62,57

Shu, F., Najita, J., Ostriker, E. et al. 1994, ApJ 429, 781

Thé, P.S., de Winter, D., Pérez, M.R. 1994, A\&AS 104, 315

Vieira, S.L.A., Pogodin, M.A., Franco, G.A.P. 1999, A\&A 345, 559 